\title{
Nasal Spray, Solution Dosage Form
}

National Cancer Institute

\section{Source}

National Cancer Institute. Nasal Spray, Solution Dosage Form. NCI Thesaurus. Code C149686.

Liquid sing le-dose or multidose preparation consisting of a solution in a container with or without a metering dose valve or in a container with a spray pump or equivalent device to create a spray, intended for nasal use. 